\title{
Unmanned Aerial Vehicle Formation Flight Using Sliding Mode Disturbance Observers
}

\author{
Dr. Galzi Damien \\ AUSY \\ France
}

\section{Introduction}

The objective of this chapter is to design a robust controller to achieve outstanding formation flight of unmanned aerial vehicles and to accurately track the desired path while facing unknown disturbances. In other words, the goal is to design the autopilot laws to enforce the desired formation by following the guidance commands. Given a non-linear mathematical model of a quadrotor (X-shaped rotorcraft), a smooth controller composed of three time-scaled nested controllers is developed using sliding mode control driven by sliding mode disturbance observer techniques based on inversion of rotorcraft dynamics and quaternions mathematical representation.

Available research efforts in multiple aircraft formation control (Innocenti et al., 2000) provide us with a variety of control techniques such as the Proportional Integral Derivative (PID) controller, Linear Quadratic Regulator (LQR) feedback controller, Adaptive controller, or control Lyapunov functions. However, these techniques have failed to address the robustness required for the tight formation to real world effects, including external disturbances, environmental aerodynamic uncertainties, end-effectors malfunctions and simulation model inaccuracies. The main limitations of the proposed techniques are the combination of accurate path tracking, adapted guidance laws for extensive formation structure capabilities, and robust formation controllers. The lack of the latter is critical for tight formation keeping in the face of leader maneuvers and unknown disturbances.

Sliding Mode (SM) control is a well-known technique used to achieve robustness and to guarantee the stability of a system (C. Edwards, \& S. Spurgeon, 1998). However it presents the inconvenience of control signal high frequency switching which is not appropriate for autopilots. Recent results in Higher Order Sliding Mode (HOSM) control theory (A. Levant, 2003) present the ability to design a continuous and even smooth control, robust to external disturbances, parametric uncertainties, end effectors failures and modeling errors, which is chattering free and provides for enhanced accuracy. Robust sliding mode control using sliding mode disturbance observer techniques allow us to consider tight formation flight where the separation distances between flying aerial vehicles are minimized. The sliding mode control technique provides the unmanned rotorcrafts with bounded, continuous output tracking for the three controllers: translation tracking, attitude tracking and speed controller for the four propellers. 
The organization of this chapter is as follow: First the unmanned vehicle is introduced and few guidelines on developing the non-linear model under quaternions representation are proposed Then the formation strategies are developed in a three dimensional environment following triangular formation strategies. Then the controller is developed for a single rotorcraft, considering that, for simplification purposes, all the rotorcrafts have the same characteristics, even though changes in mass and efficiency could easily be taken into account. Finally, simulation results will be presented within the Matlab/Simulink ${ }^{\circ}$ environment.

\section{The vehicle: X-Shaped Rotorcraft}

An example of a X-Shaped rotorcraft is the X-4 Flyer ${ }^{\circledR}$. It is propelled by four independent, fixed-pitch rotors located on the extremities of an ' $X$ ' shaped frame. This specific configuration allows a balanced distribution of thrusts around the center of mass of the vehicle. Motion is provided by independently varying the angular speed and thus the thrust of each rotor. Advantages of such a vehicle are numerous, especially for robust formation control, since soft collisions can be tolerated during flight. The major issue of the X-4-Flyer is that it is unstable by design. This makes it entirely inoperable by a human being, without the help of a fast on-board controller and motion sensors.

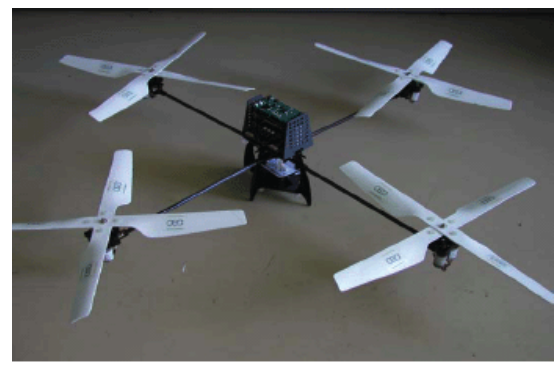

Figure 1. X-4 Flyer®
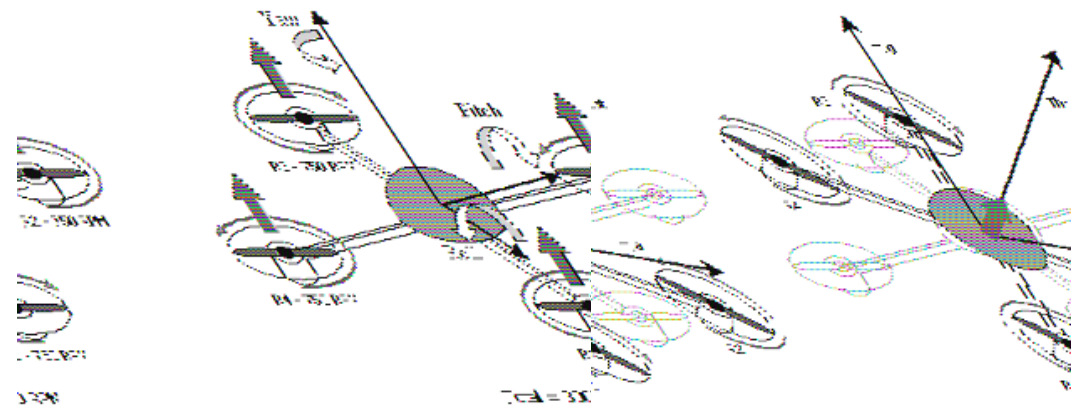

Figure 2. Hover State and Translation

The " $X$ " configuration allows rotations and translations along all three axes, but only four degrees of freedom can be controlled independently because of the coupling between these motions. Like conventional helicopters, pitch and roll rotations are coupled with longitudinal and transversal translations, respectively. 
Figure 2 shows examples of rotor speed configurations. In hover state, thrusts produced by the four propellers exactly counteract the effect of gravity. Yaw is controlled by adjusting the torque applied to each motor. The global reaction torque will cause the whole airframe to rotate about its local yaw axis. Pitch and Roll rotations can be achieved by adjusting the difference in rotor speeds within a pair of rotors rotating in the same direction. Translation is achieved by tilting the whole airframe using Pitch/Roll rotations in the direction of the desired acceleration vector, and adjusting the global thrust.

\subsection{Mathematical Model}

The frames of reference are: a fixed-to-earth frame and a local moving frame attached to the center of mass $\mathrm{O}$ of the vehicle. $\mathrm{G}$ is assumed to be Galilean. Orientation of frame A relative to frame $G$ is expressed with the rotation matrix, equation (1). The airframe orientation in space is given by the Euler angles: roll $(\phi)$, pitch $(\theta)$ and yaw $(\psi)$ with angular velocities respectively $(p, q, r)$.

$$
R_{A / G}=\left[\begin{array}{ccc}
\cos (\psi) \cos (\theta) & \sin (\phi) \sin (\theta) \cos (\psi)-\cos (\phi) \sin (\psi) & \cos (\phi) \sin (\theta) \cos (\psi)+\sin (\phi) \sin (\psi) \\
\sin (\psi) \cos (\theta) & \sin (\phi) \sin (\theta) \sin (\psi)+\cos (\phi) \sin (\psi) & \cos (\phi) \sin (\theta) \sin (\psi)-\sin (\phi) \cos (\psi) \\
-\sin (\theta) & \cos (\phi) \cos (\theta) & \cos (\phi) \cos (\theta)
\end{array}\right]
$$

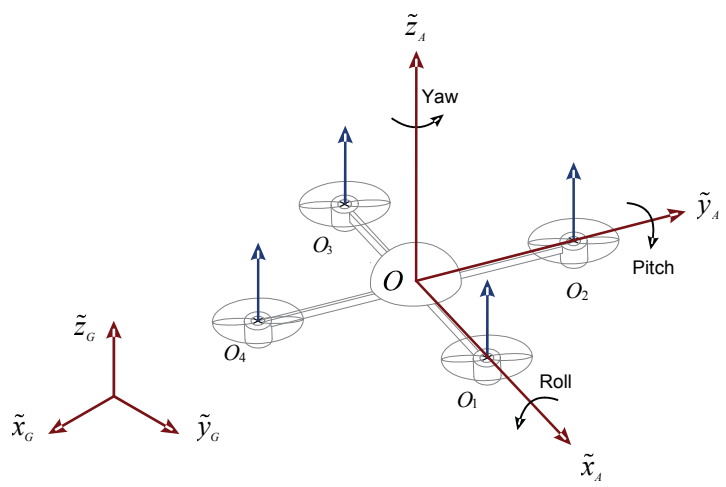

Figure 3. Frame of Reference

The following model represents an ideal case, where all variables are known or measured exactly with no measurement noise and no external disturbances. However, the model does include important realistic parameters such as aerodynamic forces and torques on airframe, propellers, viscous friction on rotor axles, and the full inertia matrix of the airframe.

Using conservation of linear and angular momentum of the airframe, conservation of angular momentum for one rotor and simplifications due to mechanical constraints of Rotor-Airframe coupling, a full realistic dynamical model of a quadrotor is proposed: Airframe linear acceleration:

$$
\left(\begin{array}{c}
\ddot{x} \\
\ddot{y} \\
\ddot{z}
\end{array}\right)_{A / G}^{A}=\dot{\widetilde{v}}_{A / G}^{G}=-g \cdot \widetilde{z}^{G}+\frac{\rho_{\text {air }}}{2 m} \cdot\left[R_{G / A}\right] \cdot\left(\begin{array}{c}
\sum_{i=1}^{4}\left(c_{r d f_{i}} \cdot \omega_{i} \cdot\left|\omega_{i}\right| \cdot \widetilde{f}_{i}^{A}\right) \\
-\left[C_{A D F}^{A}\right] \cdot\left[R_{A / G}\right] \cdot \widetilde{v}_{A / G}^{G} \cdot\left|\widetilde{v}_{A / G}^{G}{ }^{T}\right|
\end{array}\right)
$$


Airframe angular acceleration:

$$
\left(\begin{array}{l}
\dot{p} \\
\dot{q} \\
\dot{r}
\end{array}\right)_{A / G}^{A}=\dot{\widetilde{\omega}}_{A / G}^{A}=\left[I_{\text {frame/O }}^{A}\right]^{-1} \cdot\left(\begin{array}{c}
-s k\left(\widetilde{\omega}_{A / G}^{A}\right) \cdot\left[\left[I_{\text {frame/O }}^{A}\right] \cdot \widetilde{\omega}_{A / G}^{A}+\sum_{i=1}^{4}\left(j_{z_{i}} \cdot \omega_{i} \cdot \widetilde{r}_{i}^{A}\right)\right] \\
-\sum_{i=1}^{4}\left(\tau_{i}-c_{v} \cdot \omega_{i}\right) \cdot \widetilde{r}_{i}^{A} \\
+\frac{1}{2} \cdot \rho_{\text {air }} \cdot\left[-\left[C_{A D F}^{A}\right] \cdot \widetilde{\omega}_{A / G}^{A} \cdot\left|\widetilde{\omega}_{A / G}^{A}{ }^{T}\right|+\sum_{i=1}^{4}\left(c_{r d f_{i}} \cdot \omega_{i} \cdot\left|\omega_{i}\right| \cdot \widetilde{f}_{i}^{A} \times \widetilde{L}_{i}^{A}\right)\right]
\end{array}\right)
$$

$\underline{\text { Rotor angular acceleration: }}$

$$
\dot{\omega}_{i}=\frac{1}{j_{z_{i}}} \cdot\left(\tau_{i}-\frac{1}{2} \cdot \rho_{a i r} \cdot c_{r d t_{i}} \cdot \omega_{i} \cdot\left|\omega_{i}\right|-c_{v_{i}} \cdot \omega_{i}\right)
$$

$\underline{\text { Rotation matrix derivative: }}$

$$
\left[\dot{R}_{G / A}\right]=\left[R_{G / A}\right] \cdot \operatorname{sk}\left(\widetilde{\omega}_{A / G}^{A}\right)
$$

\subsection{Quaternions Representation}

A quaternion can be thought of as a complex number with 4 dimensions: 3 imaginary dimensions $i, j$ and $k$; and one real dimension, all orthogonal to one another. Each of the imaginary dimensions has a unit value of the square root of -1 . A quaternion is represented as follows:

$$
Q=q_{1} \cdot i+q_{2} \cdot j+q_{3} \cdot k+q_{4}
$$

or

$$
Q=\left[\begin{array}{l}
q_{1} \\
q_{2} \\
q_{3} \\
q_{4}
\end{array}\right]
$$

When used to describe 3D attitude or orientation, the following rule exists between quaternion coefficients and instantaneous axis $(\tilde{u})$ and angle $(\theta)$ of rotation:

$$
Q=\left[\begin{array}{l}
q_{1} \\
q_{2} \\
q_{3} \\
q_{4}
\end{array}\right]=\left[\begin{array}{c}
u_{x} \cdot \sin (\theta / 2) \\
u_{y} \cdot \sin (\theta / 2) \\
u_{z} \cdot \sin (\theta / 2) \\
\cos (\theta / 2)
\end{array}\right]
$$

It is possible to rotate vectors in $3 \mathrm{D}$ space using quaternion through the same process used with rotation matrices. The following formula shows how to rotate vector $V=\left[v_{x}, v_{y}, v_{z}\right]^{T}$ using rotation axis and angle given by quaternion $Q$

$$
\begin{gathered}
Q_{v r o t}=Q \otimes Q_{v} \otimes Q^{\prime} \\
Q_{v}=v_{x} i+v_{y} j+v_{z} k+0
\end{gathered}
$$




\section{Formation Strategies}

Formation strategies are developed to manage any size formation according to three specific schemes: "Tracking," "Placement" and "Anti-Collision." The shape of the formation is derived using "triangular" patterns depending on the number of aerial vehicle (AV).

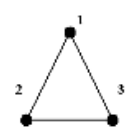

$3 \mathrm{AV}$

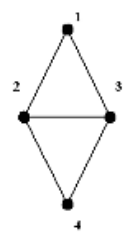

$4 \mathrm{AV}$

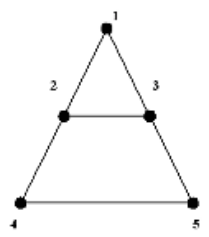

5AV

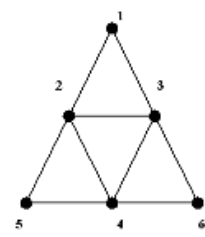

$6 \mathrm{AV}$

Figure 4. Formations Triangular Patterns

The formation strategies are designed as follows:

- The "Tracking" strategy is specific to the leader of the formation which is in this case the agent at the tip of the triangle. The task of the leader is to track the predefined path.

- The "Placement" strategy consists in positioning an agent in regards to the leader only.

- The "Anti-Collision" strategy consists in positioning an agent in regards to its lateral neighbors (set of safety distances) and to the leader.

Let us consider a formation of three agents in a Cartesian frame centered on the leader (Figure 5). The leader receives the path to follow as a function of time, while the followers receive the distances to keep from each other and to the leader. For an equilateral triangular formation pattern where the distances to keep is $d$ from each vehicle with a vertical separation of $h$, the formation commands for the followers are:

$$
\begin{gathered}
\text { "Placement": } x_{p}=x_{\text {leader }}-\sqrt{\frac{3}{4} d^{2}-h^{2}}, y_{p}=y_{\text {leader }}-\frac{d}{2}, z_{p}=z_{\text {leader }}-h \\
\text { “Anti-Collision": } x_{A}=x_{\text {leader }}-\sqrt{\frac{3}{4} d^{2}-h^{2}}, y_{A}=y_{P}+d, z_{A}=z_{\text {leader }}-h \\
\longrightarrow x
\end{gathered}
$$

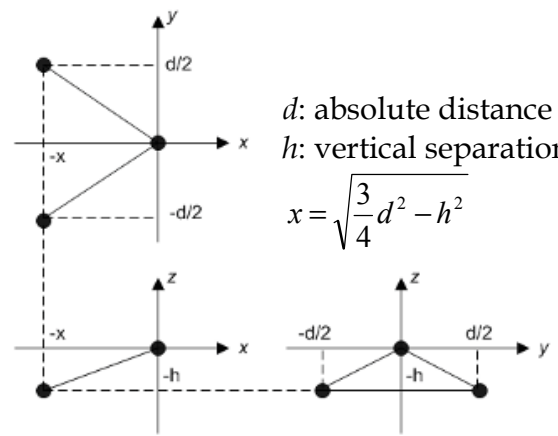

Figure 5. Three Aerial Vehicles 3D Triangular Formation Pattern 


\section{Controller Architecture}

The design of the controller follows the natural time constants of the model, which are ideally separated. A three time scale controller using three nested feedback control loops based on sliding mode driven by sliding mode observer techniques is proposed to include translation tracking controller, attitude tracking controller and speed controller for the four propellers. The control strategy is depicted in figure 6, while the architecture for each controller is depicted in figure 7 including three feedback loops and sensors to measure the real time rotorcraft state.

In loop 1 of figure 7 , the translation controller is designed to compute the instantaneous acceleration vector needed to track the reference trajectory by comparing the position commands and the actual measured positions from a Global Positioning System.

Loop 2 works in conjunction with the attitude controller to compute the body torques needed to align the rotorcraft in the direction of the desired acceleration. The desired instantaneous acceleration vector is compared to the angular velocities about the three axis of the airframe measured by gyroscopes. Attitude is derived by integration.

From the desired torque, the desired propeller velocity rates are computed using inverse kinematics (Loop 3). In the loop 4, the propeller controller stabilizes the propeller velocity rates to the commanded value compared to the measured angular rates of each rotor.

The closed-loop time constants of the three outer loop controllers have to be carefully chosen in order to guarantee convergence of each loop. Sliding mode control driven by sliding mode observer techniques is used to provide stabilization of the system dynamics and guarantee robustness; the errors will converge to the origin even in the presence of external and internal disturbances.

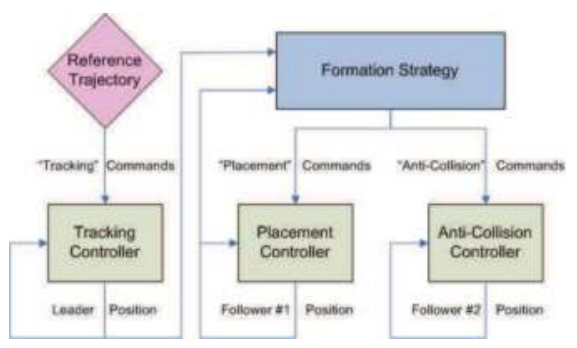

Figure 6. Formation Control Strategy

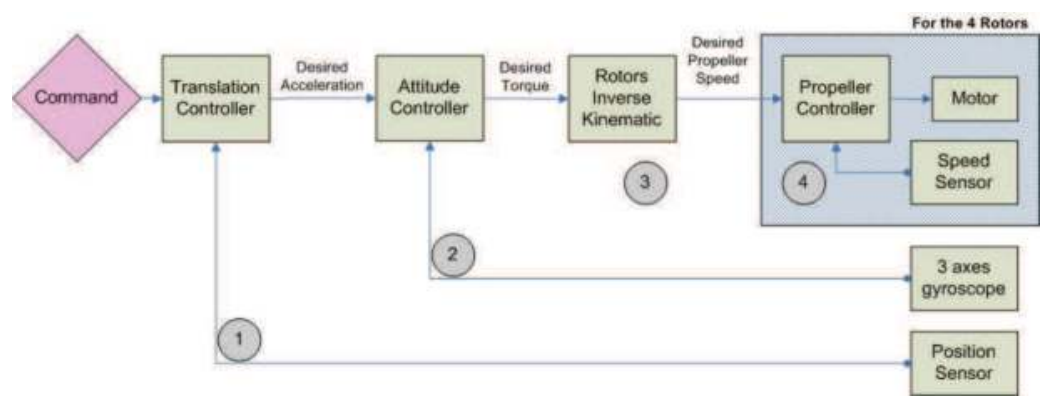

Figure 7. Flight Controller Architecture 


\section{Sliding Mode}

\subsection{Introduction to Sliding Mode}

Sliding Mode Control is a control technique that allows the user to drive a system to a desired state, referred to as sliding surface, and consists of two stages: the reaching phase and the sliding phase as shown in figure 8 . The reaching phase focuses on driving the system state to the sliding surface. The sliding phase consists of bringing the sliding variable to zero and is characterized by high-frequency, discontinuous switching of the control.

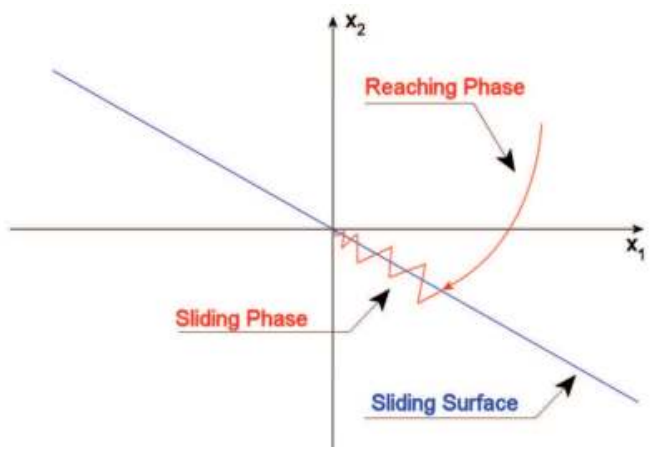

Figure 8. Sliding Mode Motion

The sliding surface is to be designed so that the system exhibits the desired behavior. The control is to be designed to assure that the system reaches the sliding surface and stays on it thereafter

Output tracking problems consider Multi Input Multi Output (MIMO) system such as the following

$$
\left\{\begin{array}{l}
\dot{x}=f(x, t)+G(x, t) u \\
y=h(x, t)
\end{array}\right.
$$

where $\quad x \in \mathfrak{R}^{n}, \quad f(x, t) \in \mathfrak{R}^{n}, \quad y \in \mathfrak{R}^{m}, \quad u \in \mathfrak{R}^{m} \quad$ and $\quad h(x, t)=\left[h_{1}, h_{2}, \ldots, h_{m}\right]^{T} \in \mathfrak{R}^{m}$, $G(x, t)=\left[g_{1}, g_{2}, \ldots, g_{m}\right]^{T} \in \mathfrak{R}^{n \times m}$ are analytic vector and matrix functions.

The system can be expressed in normal form assuming the system is completely feedback linearizable (Isidori, 1995) in a reasonable compact domain $x \in \Gamma(x)$. $L_{f}^{r_{i}-1} h_{i}$ and $L_{g_{i}}\left(L_{f}^{r_{i}-1} h_{i}\right) \quad \forall i=\overline{1, m}$ are corresponding Lie derivatives, $|E(x, t)| \neq 0, \forall x \in \Gamma$ and $\bar{r}=\left[r_{1}, r_{2}, \ldots, r_{m}\right]^{T}$ is a vector relative degree.

$$
\left[\begin{array}{c}
y_{1}^{\left(r_{1}\right)} \\
y_{2}^{\left(r_{2}\right)} \\
\ldots \\
y_{m}^{\left(r_{m}\right)}
\end{array}\right]=\left[\begin{array}{c}
L_{f}^{r_{1}} h_{1}(x, t) \\
L_{f}^{r_{2}} h_{2}(x, t) \\
\ldots \\
L_{f}^{r_{m}} h_{m}(x, t)
\end{array}\right]+E(x, t) u
$$




$$
E(x, t)=\left[\begin{array}{cccc}
L_{g_{1}}\left(L_{f}^{r_{1}-1} h_{1}\right) & L_{g_{2}}\left(L_{f}^{r_{1}-1} h_{1}\right) & \ldots & L_{g_{m}}\left(L_{f}^{r_{1}-1} h_{1}\right) \\
L_{g_{1}}\left(L_{f}^{r_{2}-1} h_{2}\right) & L_{g_{2}}\left(L_{f}^{r_{2}-1} h_{2}\right) & \ldots & L_{g_{m}}\left(L_{f}^{r_{2}-1} h_{2}\right) \\
\ldots & \ldots & \ldots & \ldots \\
L_{g_{1}}\left(L_{f}^{r_{m}-1} h_{m}\right) & L_{g_{2}}\left(L_{f}^{r_{m}-1} h_{m}\right) & \ldots & L_{g_{m}}\left(L_{f}^{r_{m}-1} h_{m}\right)
\end{array}\right]
$$

The goal of output tracking techniques is to derive the control $u$ to drive the system output $y$ to the desired trajectory $y_{c}$ (command). In other words, the goal is to drive the tracking error $e=y-y_{c}$ to zero in a finite time. The design problem is in choosing a sliding variable $\sigma$ that will drive the tracking error to zero and maintain it thereafter.

Choose the following sliding variable

$$
\sigma_{i}=e_{i}^{\left(r_{i}-1\right)}+c_{i, r_{i-2}} e_{i}^{\left(r_{i}-2\right)}+\ldots+c_{i, 1} e_{i}^{(1)}+c_{i, 0} e_{i}+c_{i,-1} e_{i,-1}
$$

where $\sigma=\left[\sigma_{1}, \sigma_{2}, \ldots, \sigma_{m}\right]^{T}, e_{i}=y_{i, c}-y_{i}, e_{i}^{(k)}=\frac{d^{k} e_{i}}{d t^{k}} \quad, \quad e_{i,-1}=\int e_{i} d t$ and $c_{i, k}>0 \in \Re$.

Taking the derivatives on both sides,

$$
\dot{\sigma}_{i}=e_{i}^{\left(r_{i}\right)}+c_{i, r_{i-2}} e_{i}^{\left(r_{i}-1\right)}+\ldots+c_{i, 1} e_{i}^{(2)}+c_{i, 0} e_{i}^{(1)}+c_{i,-1} e_{i}
$$

From equation (12),

$$
y_{i}^{\left(r_{i}\right)}=L_{f}^{r_{i}} h_{i}(x, t)+(E u)_{i}
$$

Introducing $\tilde{u}_{i}=(E u)_{i}$,

$$
e_{i}^{\left(r_{i}\right)}=y_{i, c}^{\left(r_{i}\right)}-L_{f}^{r_{i}} h_{i}(x, t)-\tilde{u}_{i}
$$

and substituting (17) into (15),

$$
\dot{\sigma}_{i}=y_{i, c}^{\left(r_{i}\right)}-L_{f}^{r_{i}} h_{i}(x, t)-\tilde{u}_{i}+c_{i, r_{i-2}} e_{i}^{\left(r_{i}-1\right)}+\ldots+c_{i, 1} e_{i}^{(2)}+c_{i, 0} e_{i}^{(1)}+c_{i,-1}
$$

Let us use the following notation

$$
\left\{\begin{array}{l}
\Psi_{i}=y_{i, c}^{\left(r_{i}\right)}-L_{f}^{r_{i}} h_{i}(x, t)+c_{i, r_{i-2}} e_{i}^{\left(r_{i}-1\right)}+\ldots+c_{i, 1} e_{i}^{(2)}+c_{i, 0} e_{i}^{(1)}+c_{i,-1} \\
\Psi_{i}=\psi_{i}^{0}+\Delta \psi_{i}
\end{array}\right.
$$

Where $\psi_{i}^{0}$ represents the known part of $\Psi_{i}$ and $\Delta \psi_{i}$ represents unknown bounded disturbances such that $\left\|\Delta \psi_{i}\right\| \leq L_{i}$. Introducing (19) into (18),

$$
\dot{\sigma}_{i}=\psi_{i}^{0}+\Delta \psi_{i}-\tilde{u}_{i}
$$

Let us design the control as

$$
\tilde{u}_{i}=\tilde{u}_{i, 0}+\tilde{u}_{i, 1}
$$


where $\tilde{u}_{i, 0}$ is designed to compensate for the known terms

$$
\tilde{u}_{i, 0}=\psi_{i}^{0}
$$

Therefore, becomes,

$$
\dot{\sigma}_{i}=\Delta \psi_{i}-\widetilde{u}_{i, 1}
$$

The control problem is to design the control $\tilde{u}_{i, 1}$ that will drive $\dot{\sigma}_{i}$ to zero.

\subsection{Traditional Sliding Mode Control}

Traditional sliding mode control is based on the analysis of Lyapunov functions to insure asymptotic stability with finite time convergence. Consider the following Lyapunov function

$$
V_{i}=\frac{1}{2} \sigma_{i}^{2}
$$

Taking the time derivative, we obtain

$$
\dot{V}_{i}=\dot{\sigma}_{i} \sigma_{i}
$$

From Lyapunov theory, we derive the following finite-time asymptotic convergence condition

$$
\dot{V}_{i} \leq-\rho_{i} \sqrt{2 V_{i}}
$$

with $\rho_{i}>0, \rho_{i} \in \Re$, which guarantees a reaching time of $t_{r, i} \leq \frac{\sqrt{2 V_{i}(0)}}{\rho_{i}}$.

Substituting (24) and (25) into (26) we obtain

$$
\begin{gathered}
\sigma_{i} \dot{\sigma}_{i} \leq-\rho_{i} \sqrt{\sigma_{i}^{2}} \\
\sigma_{i} \dot{\sigma}_{i} \leq-\rho_{i}\left|\sigma_{i}\right|
\end{gathered}
$$

From (23), we also have

$$
\begin{aligned}
& \sigma_{i} \dot{\sigma}_{i}=\sigma_{i}\left(\Delta \psi_{i}-\tilde{u}_{i, 1}\right) \\
& \sigma_{i} \dot{\sigma}_{i}=\sigma_{i} \Delta \psi_{i}-\sigma_{i} \tilde{u}_{i, 1}
\end{aligned}
$$

which can be rewritten as

$$
\sigma_{i} \dot{\sigma}_{i} \leq\left|\sigma_{i}\right|\left|\Delta \psi_{i}\right|-\sigma_{i} \tilde{u}_{i, 1}
$$


The disturbances being bounded, $\left\|\psi_{i}\right\| \leq L_{i}$, (31) becomes

$$
\sigma_{i} \dot{\sigma}_{i} \leq L_{i}\left|\sigma_{i}\right|-\sigma_{i} \tilde{u}_{i, 1}
$$

or, using the definition of the sign function

$$
\sigma_{i} \dot{\sigma}_{i} \leq\left|\sigma_{i}\right|\left[L_{i}-\tilde{u}_{i, 1} \operatorname{sign}\left(\sigma_{i}\right)\right]
$$

The control $\tilde{u}_{i, 1}$ has to satisfy the sliding mode existence condition expressed in (28). Therefore, from (33) and (28) we obtain

$$
\begin{gathered}
L_{i}-\tilde{u}_{i, 1} \operatorname{sign}\left(\sigma_{i}\right)=-\rho_{i} \\
\tilde{u}_{i, 1} \operatorname{sign}\left(\sigma_{i}\right)=\rho_{i}+L_{i}
\end{gathered}
$$

Therefore,

$$
u_{i, 1}=\left(\rho_{i}+L_{i}\right) \operatorname{sign}\left(\sigma_{i}\right)
$$

The final sliding mode control is expressed using (22), (36) and recalling that $\tilde{u}_{i}=(E u)_{i}$

$$
u=E^{-1}\left[\psi^{0}+(\rho+L) \operatorname{sign}(\sigma)\right]
$$

This traditional sliding mode control will stabilize the sliding variable on the sliding surface but is characterized by high frequency switching. Further developments in higher order sliding mode control and sliding mode control driven by sliding mode observer are employed to reduce the switching motions and accurately estimate the disturbances.

\subsection{Sliding Mode Driven by Sliding Mode Disturbance Observer}

The main idea of this technique is to design a sliding mode based observer to accurately estimate the unknown disturbance in order to compensate for it.

Introduce the auxiliary sliding variables $S_{i}$ and $Z_{i}$ such that

$$
\left\{\begin{array}{l}
s_{i}=\sigma_{i}+z_{i} \\
\dot{z}_{i}=\tilde{u}_{i, 1}-v_{i}
\end{array}\right.
$$

Therefore, the dynamic of the auxiliary sliding variable is

$$
\begin{gathered}
\dot{s}_{i}=\left(\Delta \psi_{i}-\tilde{u}_{i, 1}\right)+\left(\tilde{u}_{i, 1}-v_{i}\right) \\
\dot{s}_{i}=\Delta \psi_{i}-v_{i}
\end{gathered}
$$

As seen previously in (36), the control that will drive $S_{i}$ to zero and maintain it thereafter is

$$
v_{i}=\left(\rho_{i}+L_{i}\right) \operatorname{sign}\left(s_{i}\right)
$$


To avoid the high frequency switching motion of the control, a Low-Pass filter is introduced

$$
\hat{v}_{i}=\frac{1}{1+\beta_{i} s} v_{i}
$$

Where $\beta_{i}$ is the time constant to be chosen according to design specifications. Therefore, when the auxiliary sliding variable $S_{i}$ stabilizes at zero, (26) becomes

$$
\Delta \psi_{i}-\hat{v}_{i}=0
$$

As a result, the control $\hat{v}_{i}$ is an exact estimate of the unknown disturbances $\Delta \psi_{i}$.

Let us design the control $\tilde{u}_{i, 1}$ to stabilize $\sigma_{i}$ to zero in (23) of the form:

$$
\tilde{u}_{i, 1}=\hat{v}_{i}+K_{0, i} \sigma_{i}
$$

Where $K_{0, i}>0$ is chosen to ensure fast reaching of the sliding surface by $\sigma$, as soon as the disturbance is estimated and compensated for (i.e., the convergence of $\sigma_{i}$ cannot be faster than that of $S_{i}$ ). Therefore, the dynamic of the sliding variable is

$$
\dot{\sigma}_{i}=-K_{0, i} \sigma_{i}
$$

which satisfies the sliding mode existence condition: $\sigma_{i} \dot{\sigma}_{i}<0$.

The final sliding mode control driven by sliding mode observer is

$$
u=E^{-1}\left[\psi^{0}+\hat{v}+K_{0} \sigma\right]
$$

\section{Controller Design}

\subsection{Translation Controller}

The following equation expresses the airframe linear position, velocity and acceleration errors, respectively

$$
\begin{aligned}
& \widetilde{\varepsilon}_{p}=\widetilde{p}_{c}-\widetilde{p}_{v} \\
& \widetilde{\varepsilon}_{v}=\dot{\tilde{\varepsilon}}_{p}=\dot{\tilde{p}}_{c}-\dot{\tilde{p}} \\
& \widetilde{\varepsilon}_{\gamma}=\ddot{\widetilde{\varepsilon}}_{p}=\ddot{\tilde{p}}_{c}-\ddot{\widetilde{p}}
\end{aligned}
$$

where $\ddot{\widetilde{p}}_{c}, \dot{\tilde{p}}_{c}, \widetilde{p}_{c}$ are given by the desired trajectory commands in position, velocity and acceleration, respectively, and $\dot{\tilde{p}}, \tilde{p}$ are measured or estimated in real time by on-board sensors.

Following sliding mode observer techniques, a sliding variable of second order is designed

$$
\widetilde{\sigma}_{p}=k_{v 1} \dot{\widetilde{\varepsilon}}_{p}+k_{p 1} \widetilde{\varepsilon}_{p}+k_{i 1} \int \widetilde{\varepsilon}_{p} d t
$$


A third order pole placement is used to compute values for the coefficients $k_{v 1}, k_{p 1}$ and $k_{i 1}$. In this case, a triangular left half plane pattern pole placement is used to compute values for the coefficients, using the desired response time for position tracking.

The dynamics of the sliding variable can be expressed as

$$
\dot{\widetilde{\sigma}}_{p}=\widetilde{\psi}_{p}+\Delta \widetilde{\psi}_{p}-k_{v 1} \tilde{\gamma}
$$

where $\widetilde{\psi}_{p}$ represents the known terms,

$$
\widetilde{\psi}_{p}=k_{v 1}\left(\ddot{\widetilde{p}}_{c}+\widetilde{g}\right)+k_{p 1} \dot{\widetilde{\varepsilon}}_{p}+k_{i 1} \widetilde{\varepsilon}_{p}
$$

And $\tilde{\psi}_{p}$ the unknown bounded disturbances, $\left|\Delta \widetilde{\psi}_{p}\right| \leq L_{p}$, where $L_{p}$ is a positive constant. Therefore, the control can be expressed in a sum of control terms compensating respectively for the known and unknown terms

$$
\widetilde{\gamma}=\left(\tilde{u}_{0 p}+\tilde{u}_{1 p}\right) / k_{v 1}
$$

The disturbance observer is designed considering the following auxiliary sliding variable

$$
\begin{gathered}
\widetilde{\psi}_{p}=k_{v 1}\left(\ddot{\tilde{\vec{p}}}_{c}+\widetilde{g}\right)+k_{p 1} \dot{\widetilde{\varepsilon}}_{p}+k_{i 1} \widetilde{\varepsilon}_{p} \\
\left\{\begin{array}{l}
\widetilde{s}_{p}=\widetilde{\sigma}_{p}+\widetilde{z}_{p} \\
\dot{\widetilde{z}}_{p}=\widetilde{u}_{1 p}-\widetilde{v}_{p}
\end{array}\right.
\end{gathered}
$$

The resulting dynamics are

$$
\dot{\vec{s}}_{p}=\Delta \widetilde{\psi}_{p}-\widetilde{v}_{p}
$$

The dynamics of the auxiliary variable are completely compensated with a control of the form

$$
\widetilde{v}_{p}=\rho_{p} \operatorname{sign}\left(\widetilde{s}_{p}\right), \rho_{p}>L_{p}
$$

The high frequency switching is filtered out using the equivalent control

$$
\hat{\widetilde{v}}_{p}=\frac{\widetilde{v}_{p}}{\tau_{p} s+1}
$$

where $\tau_{p}$ is the filter time constant to be tuned.

Finally, the resulting designs of the control terms are

$$
\begin{aligned}
& \tilde{u}_{0 p}=\tilde{\psi}_{p} \\
& \tilde{u}_{1 p}=\hat{\widetilde{v}}_{p}+K_{p} \widetilde{\sigma}_{p}
\end{aligned}
$$

where $K_{p}>0$ assures exponential convergence of the sliding variable to zero. 


\subsection{Attitude Controller}

Applying inverse rotation kinematics, the desired instantaneous axis and angle of rotation are defined by computing the cross product between current thrust force vector $\widetilde{F}$ and the desired acceleration vector $\tilde{\gamma}$ normalized to unit length.

$$
\tilde{u}_{d}=\frac{\widetilde{F}}{\|\widetilde{F}\|} \times \frac{\tilde{\gamma}}{\|\widetilde{\gamma}\|}
$$

The direction of unit vector $\tilde{u}_{d}$ gives the desired axis of rotation. Therefore, the actual attitude error angle is

$$
\widetilde{\varepsilon}_{\theta}=\cos ^{-1}\left(\frac{\widetilde{F}}{\|\widetilde{F}\|} \times \frac{\widetilde{\gamma}}{\|\widetilde{\gamma}\|}\right)
$$

Equations (60) express the attitude, angular velocity and angular acceleration errors, respectively

$$
\begin{aligned}
& \widetilde{\varepsilon}_{\theta}=\widetilde{\theta}_{c}-\widetilde{\theta}=\cos ^{-1}\left(\frac{\widetilde{F}}{\|\widetilde{F}\|} \times \frac{\widetilde{\gamma}}{\left\|\widetilde{\gamma}_{d}\right\|}\right) \cdot \frac{\widetilde{u}_{u}}{\left\|\widetilde{u}_{d}\right\|} \\
& \widetilde{\varepsilon}_{w}=\dot{\widetilde{\varepsilon}}_{\theta}=\widetilde{\omega}_{c}-\widetilde{\omega}=-\widetilde{\omega} \\
& \dot{\widetilde{\varepsilon}}_{w}=\ddot{\widetilde{\varepsilon}}_{\theta}=\dot{\tilde{\omega}}_{c}-\dot{\widetilde{\omega}}=-\dot{\widetilde{\omega}}
\end{aligned}
$$

Following similar sliding mode observer techniques as presented in Section 2.D, a sliding variable of second order is designed

$$
\widetilde{\sigma}_{\theta}=k_{v 2} \dot{\widetilde{\varepsilon}}_{\theta}+k_{p 2} \widetilde{\varepsilon}_{\theta}+k_{i 2} \int \widetilde{\varepsilon}_{\theta} d t
$$

A third order pole placement is used to compute values for the coefficients $k_{v 2}, k_{p 2}$ and $k_{i 2}$. In this case, a Butterworth optimal pole placement as presented in previous work [45] is used to compute values for the coefficients, using the desired response time for attitude tracking.

Equation (62) expresses the dynamics of the sliding variable

$$
\dot{\widetilde{\sigma}}_{\theta}=\widetilde{\psi}_{\theta}+\Delta \widetilde{\psi}_{\theta}-k_{v 2} \dot{\tilde{\omega}}
$$

where $\widetilde{\psi}_{\theta}$ represents the known terms,

$$
\widetilde{\psi}_{\theta}=k_{p 2} \dot{\widetilde{\varepsilon}}_{\theta}+k_{i 2} \widetilde{\varepsilon}_{\theta}
$$

and $\Delta \tilde{\psi}_{\theta}$ the unknown bounded disturbances, $\left|\Delta \widetilde{\psi}_{\theta}\right|<L_{\theta}$, where $L_{\theta}$ is a positive constant. Once again, the control is expressed in terms of a sum of control terms compensating respectively for the known and unknown terms

$$
\dot{\widetilde{\omega}}=\left(\widetilde{u}_{0 \theta}+\tilde{u}_{1 \theta}\right) / k_{v 2}
$$


The disturbance observer is designed considering the following auxiliary sliding variable

$$
\left\{\begin{array}{l}
\widetilde{s}_{\theta}=\widetilde{\sigma}_{\theta}+\widetilde{z}_{\theta} \\
\dot{\widetilde{z}}_{\theta}=\widetilde{u}_{1 \theta}-\widetilde{v}_{\theta}
\end{array}\right.
$$

The resulting dynamics are

$$
\dot{\widetilde{s}}_{\theta}=\Delta \widetilde{\psi}_{\theta}-\widetilde{v}_{\theta}
$$

The dynamics of the auxiliary variable are completely compensated for with control of the form

$$
\widetilde{v}_{\theta}=\rho_{\theta} \operatorname{sign}\left(\widetilde{s}_{\theta}\right), \rho_{\theta} \gg L_{\theta}
$$

The high frequency switching is filtered out using the equivalent control

$$
\hat{\widetilde{v}}_{\theta}=\frac{\widetilde{v}_{\theta}}{\tau_{\theta} s+1}
$$

where $\tau_{\theta}$ is the filter time constant to be tuned. Finally, the resulting design of the control terms is

$$
\begin{aligned}
& \tilde{u}_{0 \theta}=\tilde{\psi}_{\theta} \\
& \tilde{u}_{1 \theta}=\hat{\widetilde{v}}_{\theta}+K_{\theta} \tilde{\sigma}_{\theta}
\end{aligned}
$$

where $K_{\theta}>0$ assure exponential convergence of the sliding variable to zero.

\subsection{Propeller Controller}

The speed of each rotor $\left(w_{i}, i=1 \ldots 4\right)$ is computed using inverse dynamics as presented in and compared to the desired speed $\left(w_{i d}, i=1 \ldots 4\right)$ corresponding to the desired linear $(\tilde{\gamma})$ and angular $(\dot{\tilde{\omega}})$ accelerations. The necessary torques to apply to each electric motor are then computed in order to achieve these desired speeds.

The angular velocity error of each rotor is defined as

$$
\begin{aligned}
& \varepsilon_{\omega i}=\omega_{i d}-\omega_{i} \\
& \dot{\varepsilon}_{\omega i}=\dot{\omega}_{i d}-\dot{\omega}_{i}=-\dot{\omega}_{i}
\end{aligned}
$$

Using the same sliding mode observer techniques as for the previous two controllers, the sliding variable

$$
\sigma_{\omega i}=k_{p 3} \varepsilon_{\omega i}+k_{i 3} \int \varepsilon_{\omega i} d t
$$

A classical second order placement is used, with the desired response time, to define $k_{p 3}$ and $k_{i 3}$. The dynamics of the sliding variable are expressed as

$$
\dot{\sigma}_{\omega i}=\psi_{\omega i}+\Delta \psi_{\omega i}-k_{p 3} \dot{\omega}_{i}
$$


where $\psi_{\omega i}$ represents the known terms

$$
\psi_{\omega i}=k_{i 3} \varepsilon_{\omega i}
$$

and $\Delta \psi_{\omega i}$ the unknown bounded disturbances, $\Delta \psi_{\omega i}<L_{\omega i}$, where $L_{\omega i}$ is a positive constant. Once again, the control is expressed in terms of a sum of control terms compensating respectively for the known and unknown terms

$$
\dot{\omega}_{i}=\left(u_{0 \omega i}+u_{1 \omega i}\right) / k_{p 3}
$$

The disturbance observer is designed considering the auxiliary sliding variable

$$
\left\{\begin{array}{l}
s_{\omega i}=\sigma_{\omega i}+z_{\omega i} \\
\dot{z}_{\omega i}=u_{1 \omega i}-v_{\omega i}
\end{array}\right.
$$

The resulting dynamics are

$$
\dot{s}_{\omega i}=\Delta \psi_{\omega i}-v_{\omega i}
$$

The dynamics of the auxiliary variable are completely compensated for with control of the form

$$
v_{\omega i}=\rho_{\omega i} \operatorname{sign}\left(s_{\omega i}\right), \rho_{\omega i} \gg L_{\omega i}
$$

The high frequency switching is filtered out using the equivalent control

$$
\hat{v}_{\omega i}=\frac{v_{\omega i}}{\tau_{\omega i} s+1}
$$

where $\tau_{w i}$ is the filter time constant to be tuned. Finally, the resulting design of the control terms is

$$
\begin{aligned}
& u_{0 \omega i}=\psi_{\omega i} \\
& u_{1 \omega i}=\hat{v}_{\omega i}+K_{\omega i} \sigma_{\omega i}
\end{aligned}
$$

where $K_{w i}>0$ assures exponential convergence of the sliding variable to zero.

\section{Simulation Results}

The following figures are the simulation results for the formation of three X-4 Flyer flying in a close formation in the face of simulated wind gusts acting upon each rotorcraft (modeled as offset on the velocity vector in frame G). The formation is to fly in a triangular pattern with an absolute distance of three meters $(d=3 \mathrm{~m})$ between each rotorcraft and a vertical separation of one meter to the leader. The trajectory to follow is a sinusoid at a constant elevation of 2 meters and a constant longitudinal velocity of $2 \mathrm{~m} / \mathrm{s}$. Note that the first follower will stay at the initial position for one second following take off of the leader and then follow the "Placement" commands. The second follower will stay at the initial position for two seconds following the take off of the leader (or one second following the take off of 
the first follower) and then follow the "Anti-Collision" commands. The simulation time is 10sec with a computing frequency of a $1000 \mathrm{~Hz}$.

\subsection{Leader "Path Tracking"}

The following figures represent the "Path Tracking" simulation results for the leader of the formation. The characteristics of the X-4 Flyer, such as position, velocity, angular velocity and propeller angular velocity are illustrated, as well as the control torques and the HOSM controller variables.
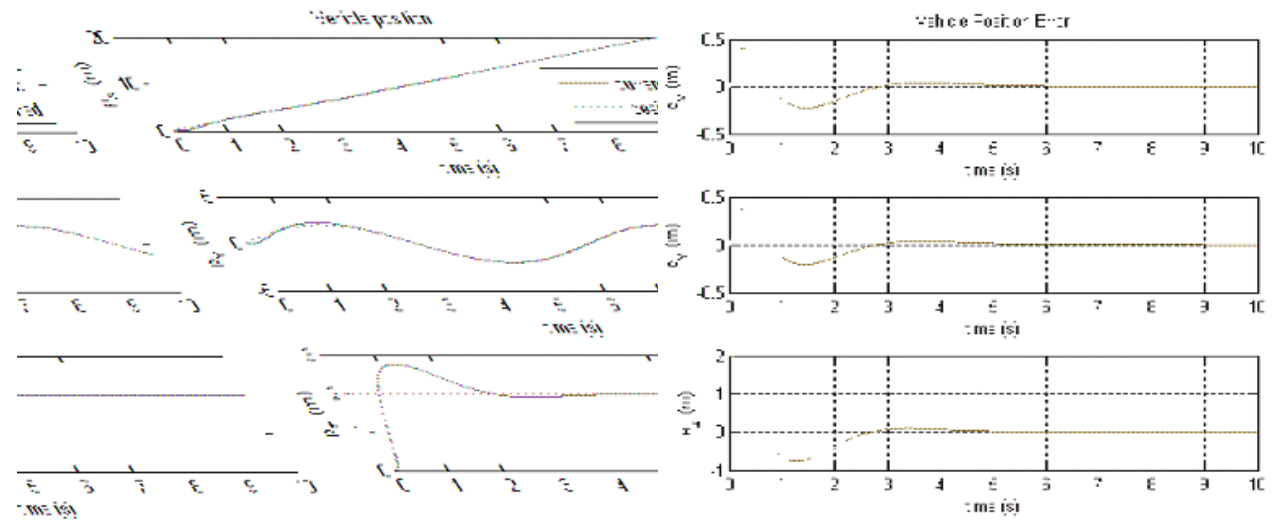

Figure 9. Leader Position and Position Error versus Time

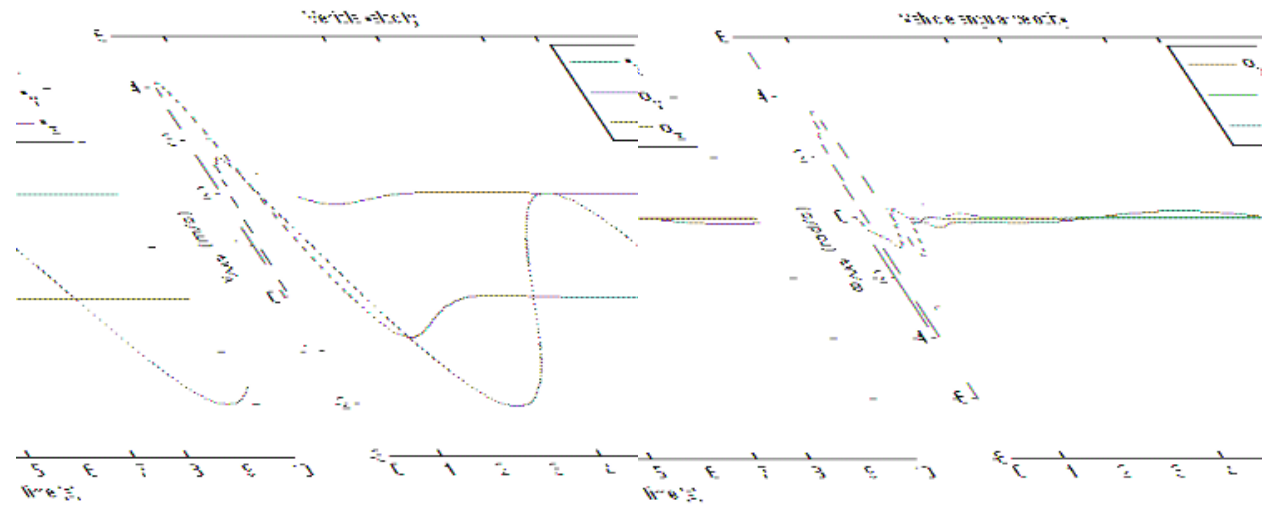

Figure 10. Leader Velocity and Angular Velocity versus Time 

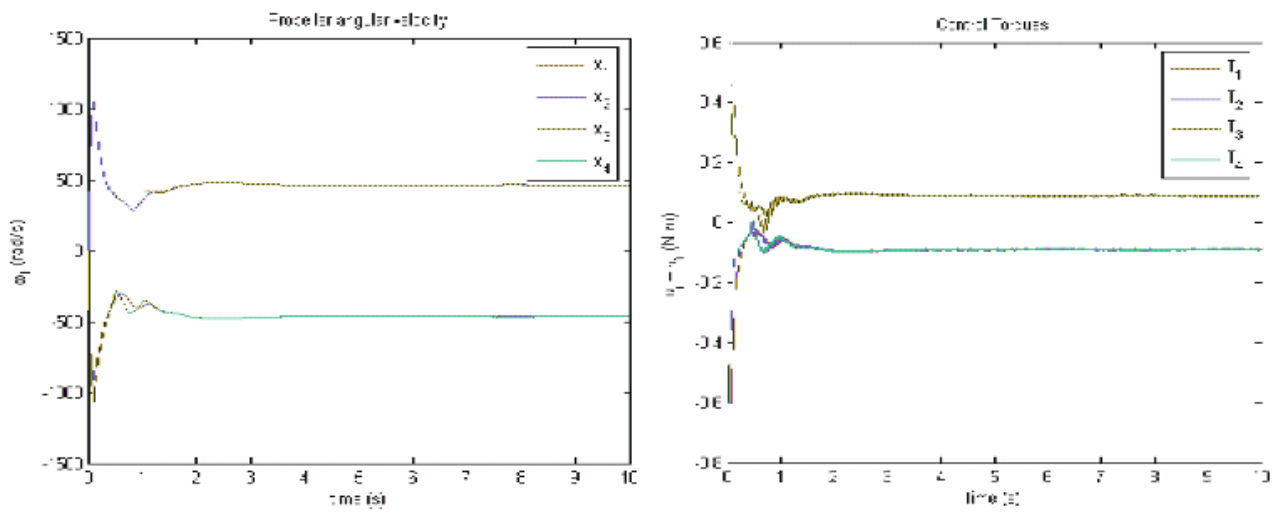

Figure 11. Leader Propeller Angular Velocity and Control Torques versus Time
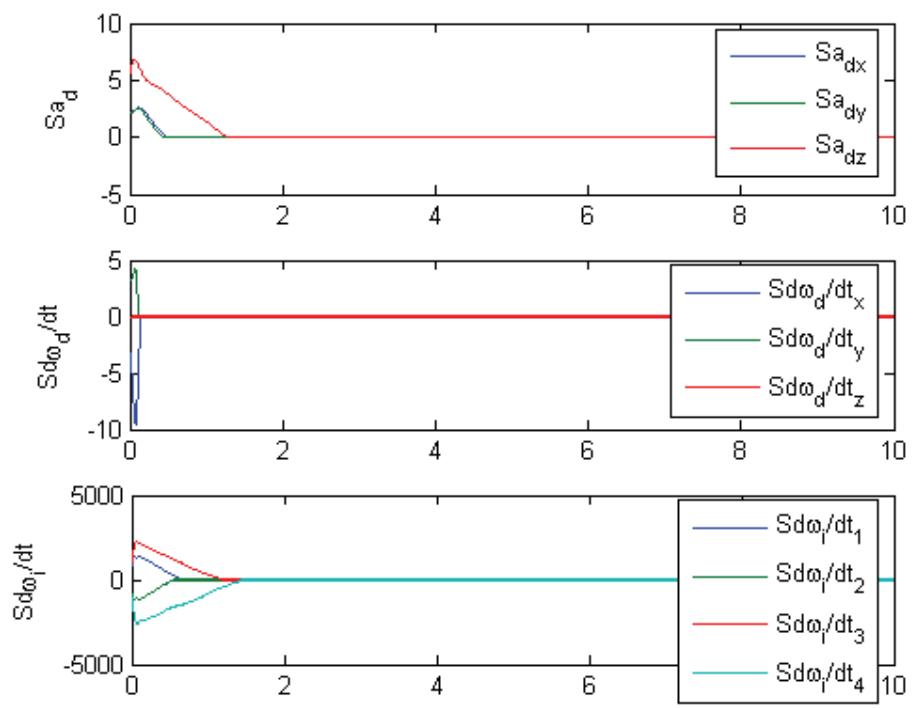

Figure 12. Leader Auxiliary Sliding Variables versus Time

The leader achieves perfect tracking of the desired path in a smooth and robust manner. The position errors in 3 reach zero in finite time. The unknown disturbances are estimated and completely compensated for using robust HOSM techniques.

\subsection{First Follower "Placement"}

The following figures represent the "Placement" simulation results for the first follower. The characteristics of the X-4 Flyer, such as position, velocity, angular velocity and propeller angular velocity are illustrated, as well as the control torques and the HOSM controller variables. 

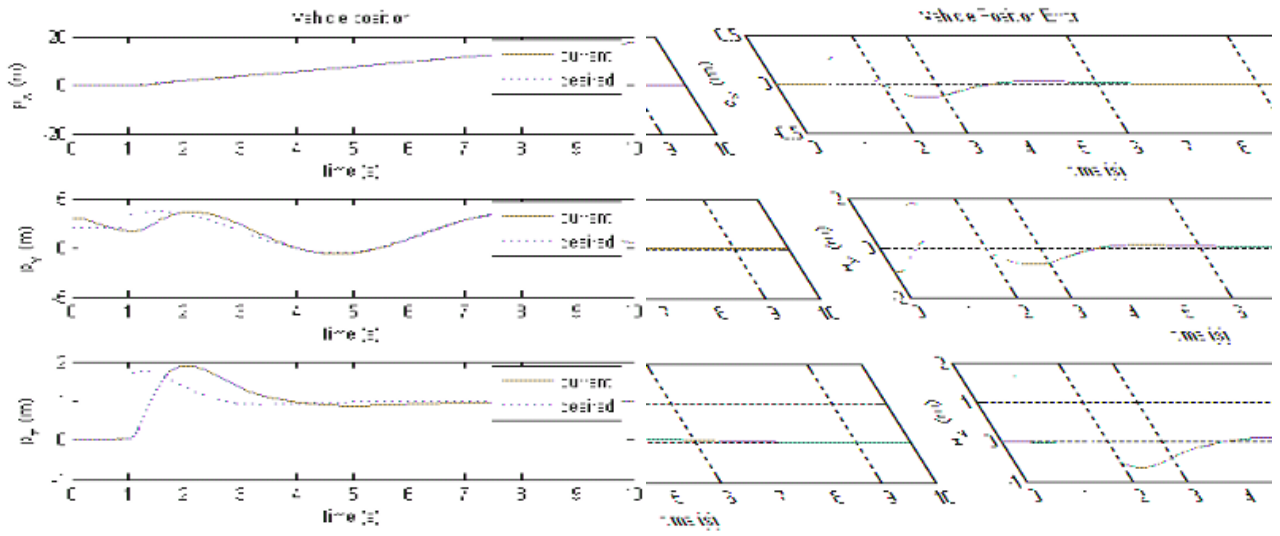

Figure 13. First Follower Position and Position Error versus Time

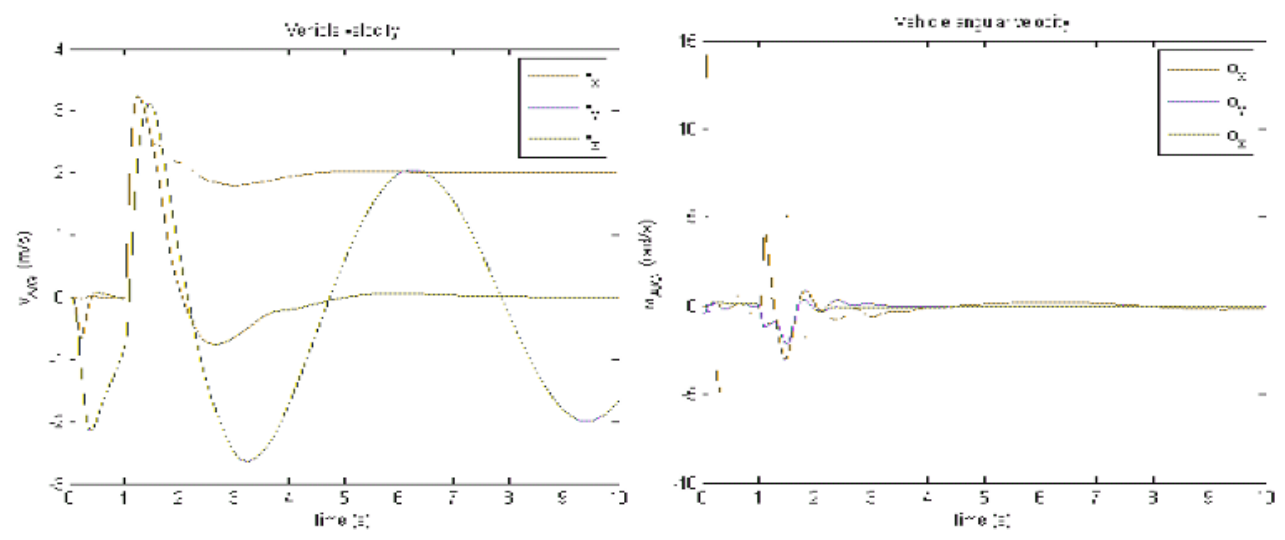

Figure 14. First Follower Velocity and Angular Velocity versus Time

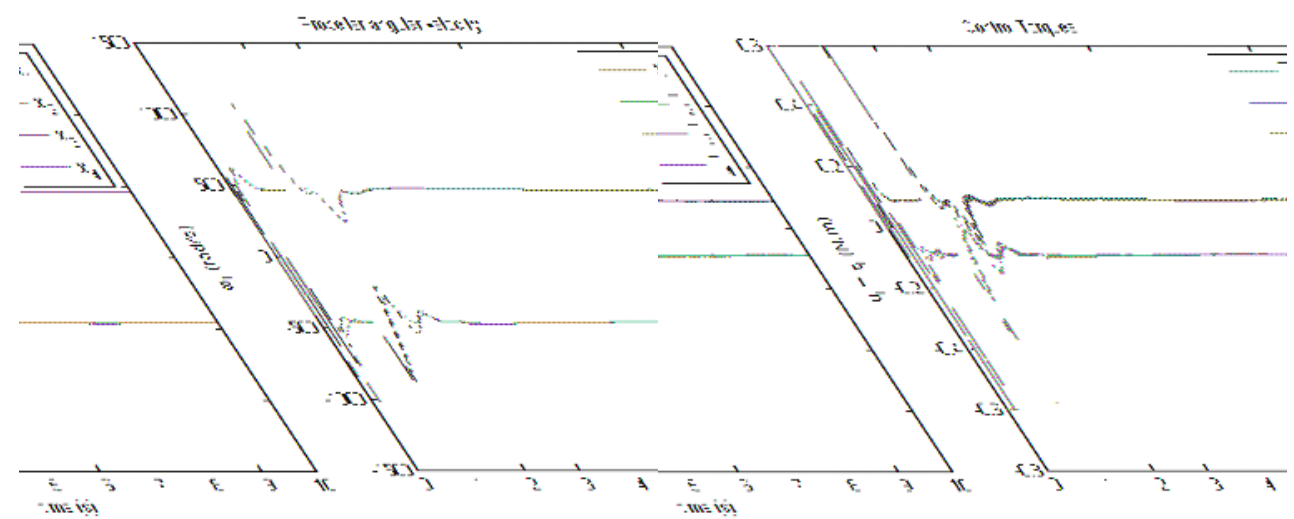

Figure 15. First Follower Propeller Angular Velocity and Control Torques versus Time 

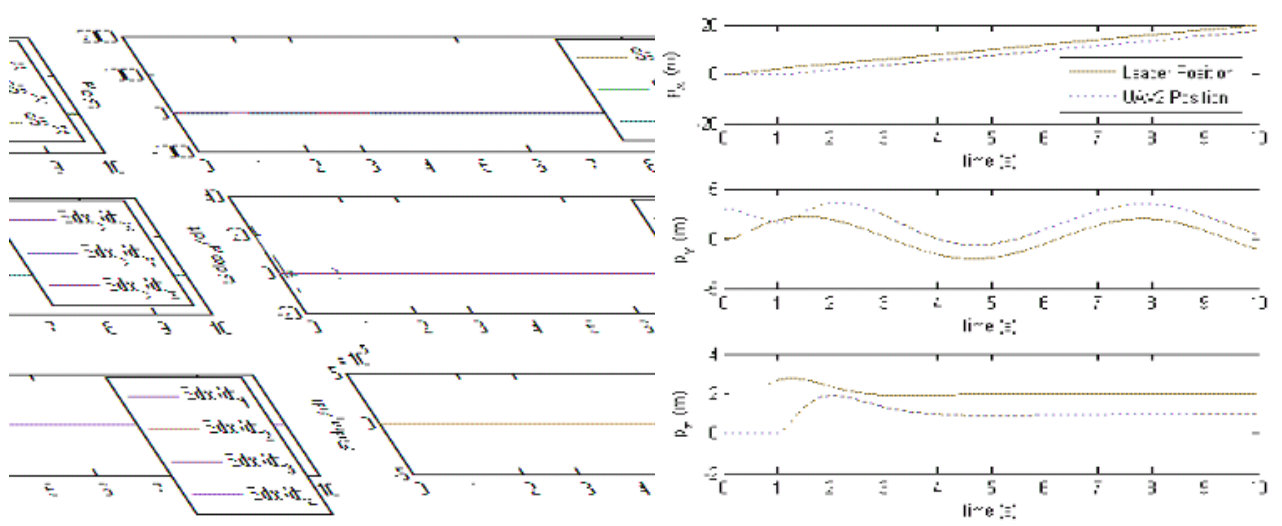

Figure 16. First Follower Auxiliary Sliding Variables and Separation versus Time

After staying at the initial position for the first second, the rotorcraft initiates the formation following the "Placement" commands. The rotorcraft achieves perfect positioning after a smooth transient, the position errors reach zero in finite time. The disturbances are estimated and fully compensated for, via robust HOSM.

\subsection{Second Follower "Anti-Collision"}

The following figures represent the "Anti-Collision" simulation results for the second follower. The characteristics of the X-4 Flyer, such as position, velocity, angular velocity and propeller angular velocity are illustrated, as well as the control torques and the HOSM controller variables.
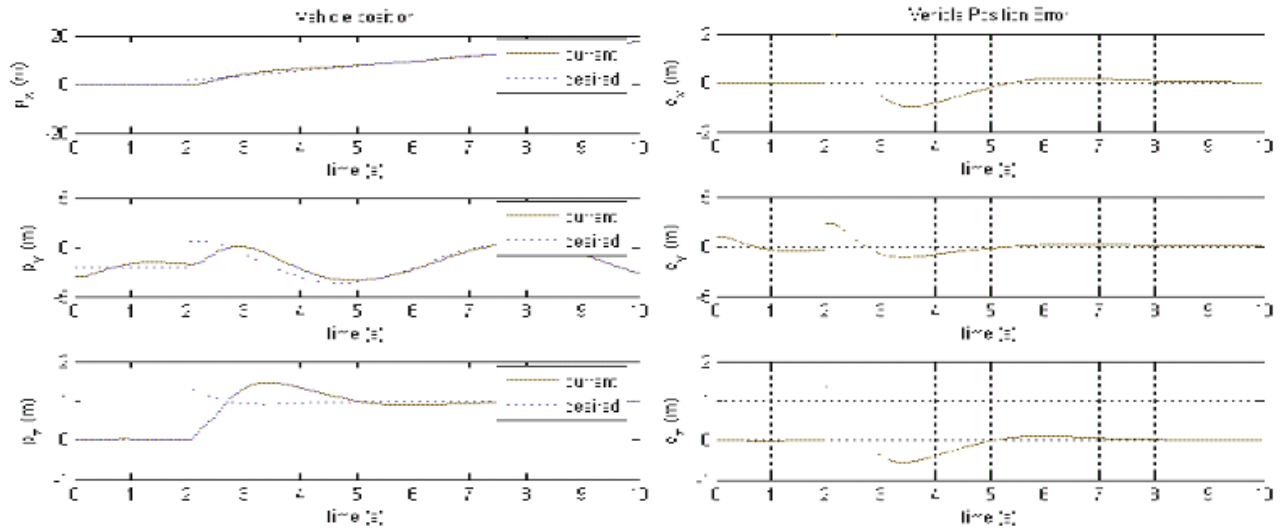

Figure 17. Second Follower Position and Position Error versus Time 


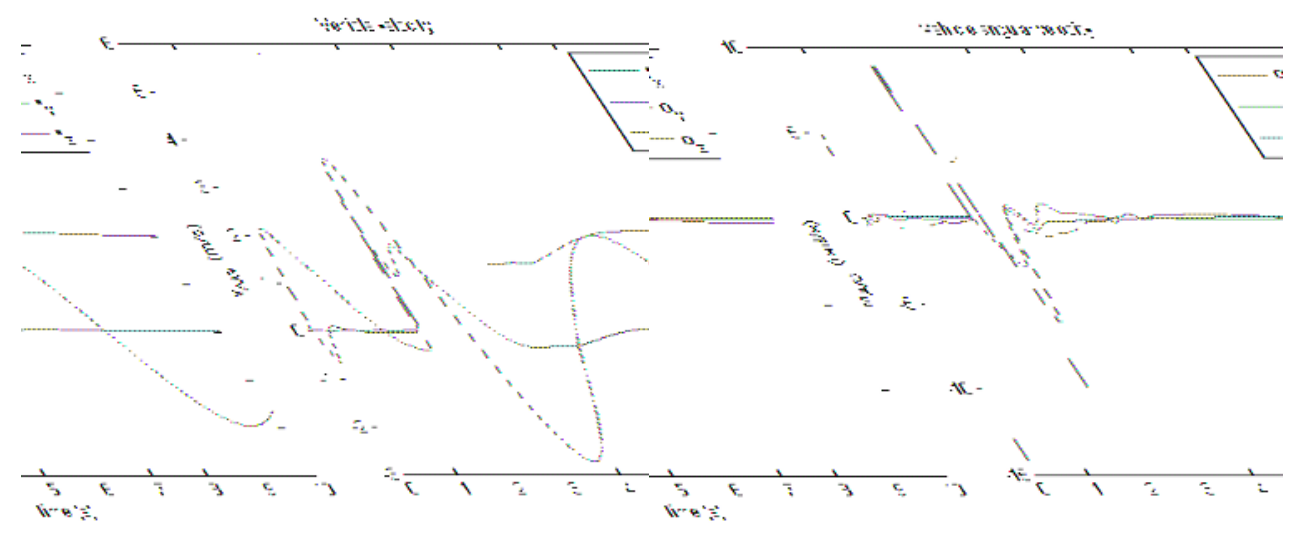

Figure 18. Second Follower Velocity and Angular Velocity versus Time

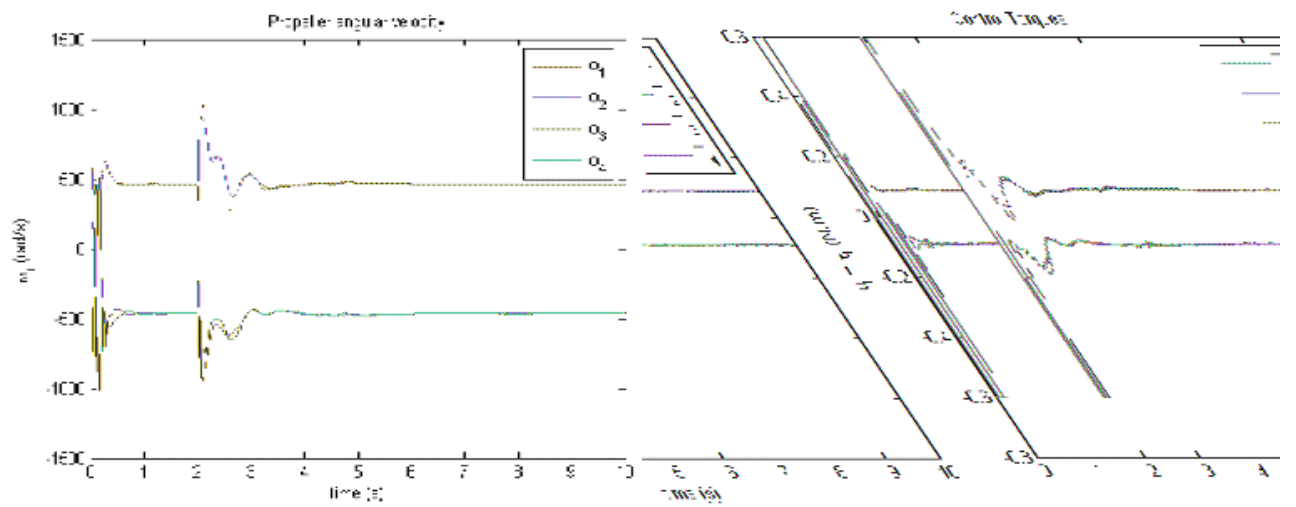

Figure 19. Second Follower Propeller Angular Velocity and Control Torques versus Time
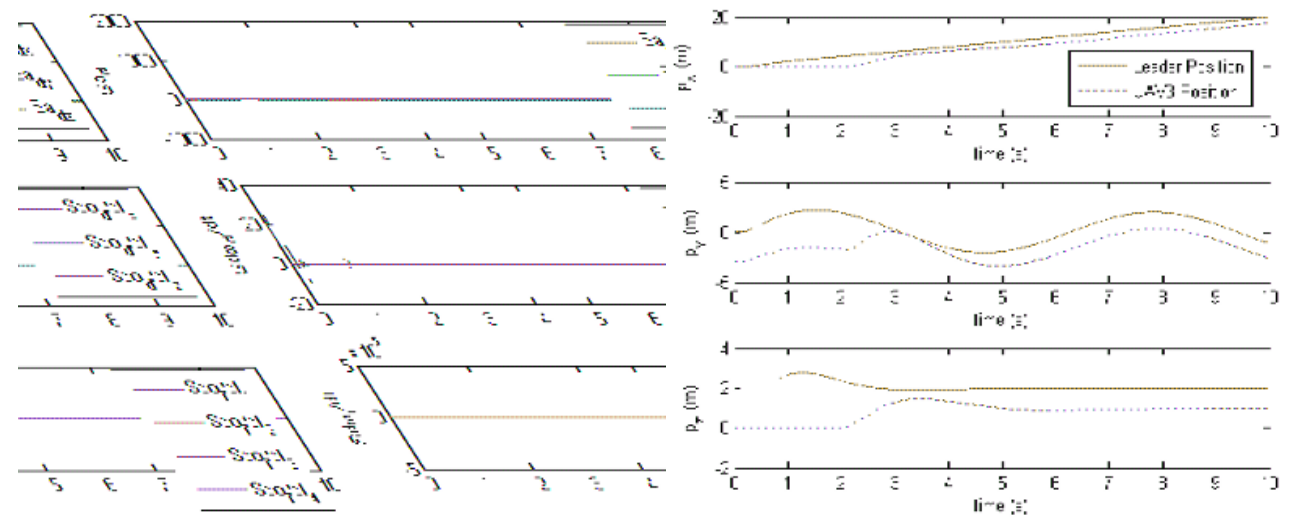

Figure 20. Second Follower Auxiliary Sliding Variables and Separation versus Time 
Finally, the second follower initiates the "Anti-Collision" strategy after two seconds of delay at initial condition. The rotorcraft achieves perfect positioning after a short transient. The rotorcraft has to "catch up" with the rest of the formation and, therefore, must utilize all available resources to rapidly reach the desired position, which can be seen by the saturation of the torques. Once again, the disturbances are estimated and fully compensated for via robust HOSM.

\subsection{Formation tracking}

The rotorcrafts initiate and keep the desired formation on the right path after a certain transient. The absolute distance between rotorcrafts reaches exactly the desired separation. The desired triangular formation following the prescribed sinusoidal path is represented in the following figures.

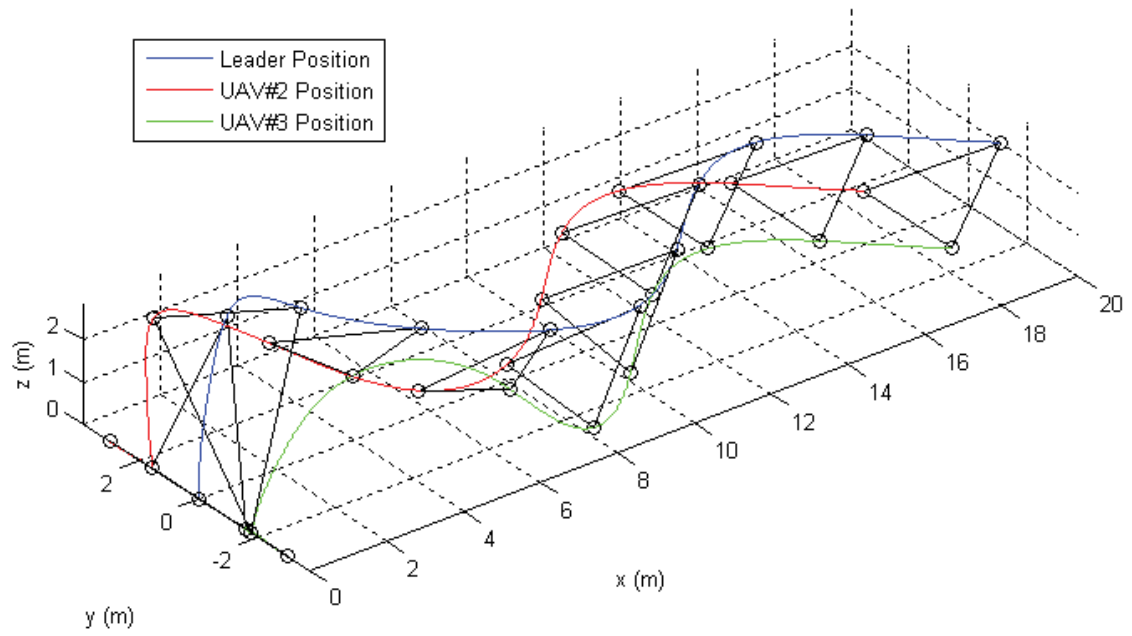

Figure 21. Three X-4 Flyer Formation Flight

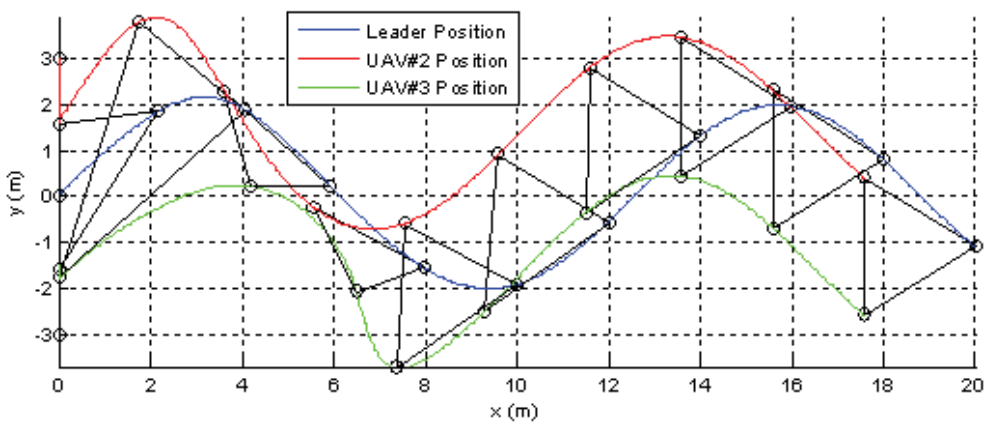

Figure 22. Three X-4 Flyer Formation Flight X-Y Projection 


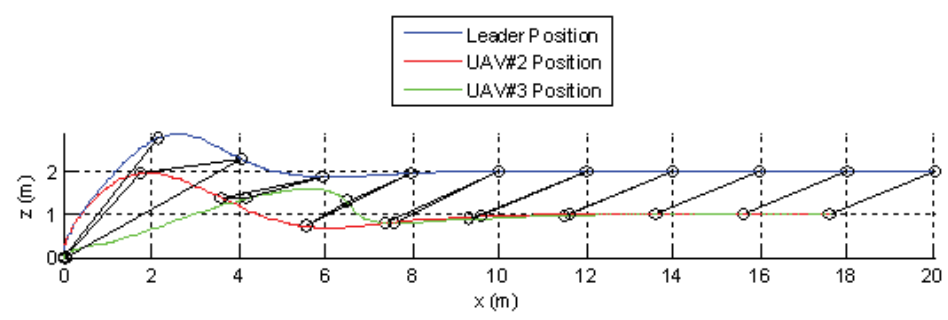

Figure 23. Three X-4 Flyer Formation Flight X-Z Projection

\subsection{Actuator Malfunction}

One of the propeller of the first follower is not working properly; 4 seconds into the simulation, the propeller loses efficiency and the motor torque is dropped by $50 \%$. In order to keep the rotorcraft balanced, the propeller angular velocity must be restored as fast as possible by adjusting the applied torque. 32 shows that the controller doubles the appropriate motor torques to compensate for the loss of efficiency quickly, restoring the angular velocity. The flying formation is not affected by the actuator malfunction, thus proving the robustness of the proposed HOSM techniques.
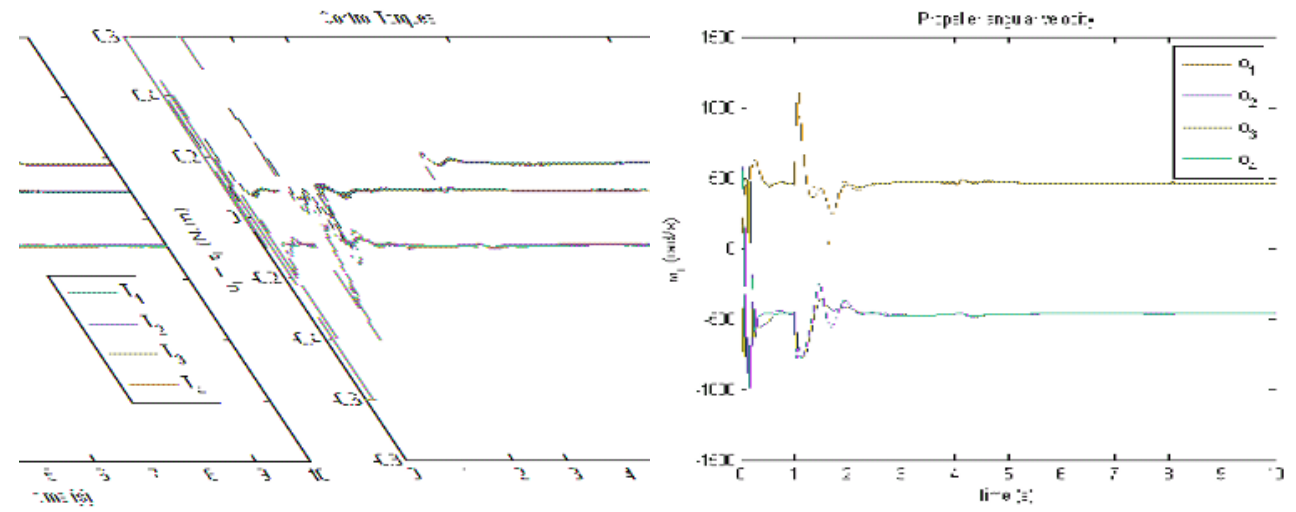

Figure 24. Compensating Torques and Restored Angular Velocity

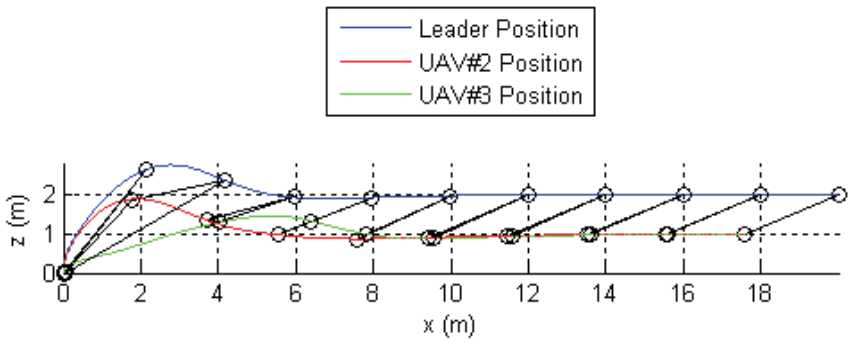

Figure 25. Formation Flying with Actuator Malfunction X-Z projection 


\section{Conclusion}

In this chapter, formation strategies are applied to the close formation flight of three XShaped rotorcrafts in a full 3D environment. Robust formation tracking is achieved via sliding mode driven by sliding mode disturbance observer techniques. Simulation results show that SMDO controllers exhibit smooth and continuous controls in the presence of unknown bounded disturbances and actuator malfunction. The controls provide finite time convergence of the tracking errors to zero after a short transient corresponding to the initialization of the formation. Perfect close formation is achieved in the face of leader maneuvers and unknown disturbances, which proves the effectiveness of the proposed techniques.

Possible extensions of the research include the implementation of actual formation flight scenarios with leader reassignment and formation reconfiguration. Also, an embedded collision avoidance algorithm could be added to each aerial vehicle in the formation. Results using potential field techniques are interesting and available in the literature.

\section{List of Symbols}

\begin{tabular}{|c|c|}
\hline $\begin{array}{l}\tilde{x}_{A}, \tilde{y}_{A}, \tilde{z}_{A} \\
\tilde{V}^{A}\end{array}$ & $\begin{array}{l}\text { Unit vectors respectively along } \mathrm{X}_{\mathrm{A}}, \mathrm{Y}_{\mathrm{A}} \text { and } \mathrm{Z}_{\mathrm{A}} \text { axes of frame } \mathrm{A} \\
\text { Vector } \mathrm{V} \text { expressed in frame } \mathrm{A}\end{array}$ \\
\hline$\dot{\tilde{V}}^{A}$ & Derivative of vector $\mathrm{V}$ expressed in frame $\mathrm{A}$ \\
\hline$\left[M^{A}\right]$ & Matrix $\mathrm{M}$ expressed in frame $\mathrm{A}$ \\
\hline$\tilde{F}_{B_{1} \rightarrow B_{2}}^{A}$ & Force vector exerted from body $B_{1}$ to body $B_{2}$ expressed in frame $A$ \\
\hline$\tilde{\tau}_{B_{1} \rightarrow B_{2}}^{A}$ & Torque vector exerted from body $B_{1}$ to body $B_{2}$ expressed in frame $A$ \\
\hline $\operatorname{sk}\left(\tilde{V}^{A}\right)$ & Skew matrix of vector $\mathrm{V}$ expressed in frame $\mathrm{A}$ (used for cross product) \\
\hline$(\phi, \theta, \psi)$ & Euler angles \\
\hline$(p, q, r)$ & Angular velocities \\
\hline$\left[R_{G / A}\right]$ & Rotation matrix to go from frame A to frame $G$ \\
\hline$D$ & Diameter of propeller $(\mathrm{m})$ \\
\hline$\left[I_{\text {frame } O O}^{A}\right]$ & Inertia matrix of airframe expressed in frame $\mathrm{A}$ at point $\mathrm{O}\left(\mathrm{kg} \cdot \mathrm{m}^{2}\right)$ \\
\hline$\left[I_{\text {rotor } / O_{i}}^{A}\right]$ & Inertia matrix of rotor ' $\mathrm{i}$ ' expressed in frame $A$ at point $\mathrm{O}_{\mathrm{i}}\left(\mathrm{kg} \cdot \mathrm{m}^{2}\right)$ \\
\hline$\tilde{v}_{A / G}^{G}$ & Linear velocity vector of frame A relative to frame $G$ (ground) $\left({\left.\mathrm{m} . \mathrm{s}^{-1}\right)}^{-1}\right.$ \\
\hline$\tilde{\omega}_{A / G}^{A}=(p, q, r)^{T}$ & Angular velocity vector of frame A relative to frame G (rad.s $\left.{ }^{-1}\right)$ \\
\hline$\tilde{\omega}_{R_{i} / A}^{A}$ & Angular velocity vector of rotor ' $\mathrm{i}$ ' relative to frame A (rad.s $\left.{ }^{-1}\right)$ \\
\hline$\omega_{i}$ & Angular velocity of rotor ' $\mathrm{i}$ ' with respect to frame A (rad.s-1) \\
\hline$\tilde{r}_{i}^{A}$ & Axis of rotation (unit vector) of rotor ' $i$ ' expressed in frame A. \\
\hline$\tilde{f}_{i}^{A}$ & Direction of thrust produced by rotor ' $i$ ' expressed in frame A. \\
\hline$\tau_{i}$ & Torque produced by motor ' $\mathrm{i}$ ' on rotor ' $\mathrm{i}$ ' $\left(\mathrm{N} \cdot \mathrm{m}=\mathrm{kg} \cdot \mathrm{m}^{2} \cdot \mathrm{s}^{-2}\right)$ \\
\hline
\end{tabular}


$F_{i}$

$\tilde{L}_{i}^{A}$

$\tilde{\tau}_{F_{i}}^{A}$

$\left[C_{A D F}^{A}\right]$

$\left[C_{A D T}^{A}\right]$

$\left[C_{R D T_{i}}^{A}\right]$

$\left[C_{V_{i}}^{A}\right]$

$c_{r d f_{i}}$

$c_{v_{i}}$

$\tilde{p}_{c}, \dot{\tilde{p}}_{c}, \ddot{\tilde{p}}_{c}$

$\tilde{\varepsilon}_{p}, \tilde{\varepsilon}_{v}, \tilde{\varepsilon}_{\gamma}$

$k_{v 1}, k_{p 1}, k_{i 1}$

$k_{v 2}, k_{p 2}, k_{i 2}$

$k_{p 3}, k_{i 3}$

$\tilde{F}_{i}$

$\tilde{F}$

Thrust force produced by rotor ' $\mathrm{i}$ ' expressed in frame A ( $\left.\mathrm{N}=\mathrm{kg} \cdot \mathrm{m} \cdot \mathrm{s}^{-2}\right)$

Vector $\overrightarrow{O_{i} O}$ between rotor and center of mass expressed in frame A (m)

Torque produced by rotor ' $\mathrm{i}$ ' on airframe expressed in frame A (N.m)

Drag force coefficient matrix of airframe expressed in frame $\mathrm{A}\left(\mathrm{m}^{2}\right)$

Drag torque coefficient matrix of airframe expressed in frame A $\left(\mathrm{m}^{5}\right)$

Drag torque coefficient matrix of rotor ' $i$ ' expressed in frame A $\left(\mathrm{m}^{5}\right)$

Viscous coefficient matrix of rotor ' $\mathrm{i}$ ' expressed in frame A $\left(\mathrm{kg} \cdot \mathrm{m}^{2} \cdot \mathrm{s}^{-1}\right)$

Drag torque coefficient of rotor ' $\mathrm{i}$ ' along its axis of rotation $\left(\mathrm{m}^{5}\right)$

Viscous friction coefficient of rotor ' $\mathrm{i}$ ' $\left(\mathrm{kg} \cdot \mathrm{m}^{2} \cdot \mathrm{s}^{-1}\right)$

Commanded trajectory position, velocity and acceleration

Position, velocity and acceleration error vectors respectively

Position controller feedback coefficients

Attitude controller feedback coefficients

Propeller controller feedback coefficients

Thrust force produced by each rotor expressed in frame A (N)

Current total thrust force expressed in frame $\mathrm{G}(\mathrm{N})$

\section{References}

C. Edwards, \& S. Spurgeon, Sliding Mode Control, Taylor \& Francis, Bristol, PA, 1998.

M. Innocenti, L. Pollini, \& F. Guilietti, Autonomous Formation Flight, IEEE Controls System Magazine, Vol. 20, No. 6, 2000, pp. 34-44.

A. Isidori, Nonlinear Control Systems, Springer-Verlag, London, 3rd Ed., 1995.

A. Levant, Higher-Order Sliding Modes, Differentiation and Output-Feedback Control, International journal of Control, 76 (9/10), pp. 924-941, 2003. 


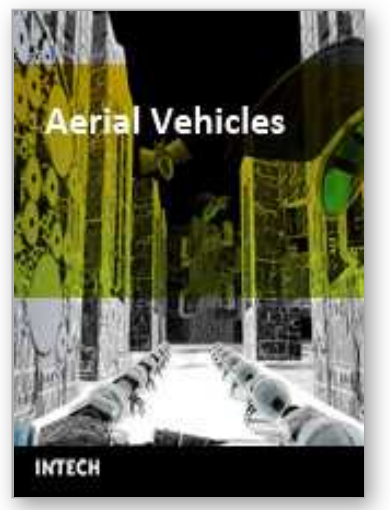

\author{
Aerial Vehicles \\ Edited by Thanh Mung Lam
}

ISBN 978-953-7619-41-1

Hard cover, 320 pages

Publisher InTech

Published online 01, January, 2009

Published in print edition January, 2009

This book contains 35 chapters written by experts in developing techniques for making aerial vehicles more intelligent, more reliable, more flexible in use, and safer in operation. It will also serve as an inspiration for further improvement of the design and application of aeral vehicles. The advanced techniques and research described here may also be applicable to other high-tech areas such as robotics, avionics, vetronics, and space.

\title{
How to reference
}

In order to correctly reference this scholarly work, feel free to copy and paste the following:

Galzi Damien (2009). Unmanned Aerial Vehicle Formation Flight Using Sliding Mode Disturbance Observers, Aerial Vehicles, Thanh Mung Lam (Ed.), ISBN: 978-953-7619-41-1, InTech, Available from: http://www.intechopen.com/books/aerial_vehicles/unmanned_aerial_vehicle_formation_flight_using_sliding_m ode_disturbance_observers

\section{INTECH}

open science | open minds

\section{InTech Europe}

University Campus STeP Ri

Slavka Krautzeka 83/A

51000 Rijeka, Croatia

Phone: +385 (51) 770447

Fax: +385 (51) 686166

www.intechopen.com

\section{InTech China}

Unit 405, Office Block, Hotel Equatorial Shanghai No.65, Yan An Road (West), Shanghai, 200040, China 中国上海市延安西路65号上海国际贵都大饭店办公楼 405 单元 Phone: $+86-21-62489820$

Fax: +86-21-62489821 
(C) 2009 The Author(s). Licensee IntechOpen. This chapter is distributed under the terms of the Creative Commons Attribution-NonCommercialShareAlike-3.0 License, which permits use, distribution and reproduction for non-commercial purposes, provided the original is properly cited and derivative works building on this content are distributed under the same license. 